\title{
Enhancement of Maximal Ratio Combiner over Log-Normal Fading Channel using Firefly Algorithm
}

\author{
${ }^{1}$ Abolade, R.O., ${ }^{2}$ Ojo, S.I, ${ }^{3}$ Salami, H.T., ${ }^{4}$ Adeyemo, Z.K., ${ }^{5}$ Akintoye, N.O. \\ 1,2,4Electronic and Electrical Engineering Department, Ladoke Akintola University of Technology, \\ Ogbomoso, Nigeria \\ ${ }^{3}$ Department of Electronic and Computer Engineering, Lagos State University, Epe Campus, Epe, Nigeria \\ ${ }^{5}$ Electronic and Electrical Engineering Department, the Polytechnic Ibadan, Ibadan, Nigeria \\ roabolade@lautech.edu.ng; samyem141@gmail.com; siojo85@pgschool.lautech.edu.ng; \\ zkadeyemo@lautech.edu.ng; akintoyenafiu@gmail.com
}

\begin{abstract}
Wireless communication system has a great impact in the world of telecommunication and is expected to be a leading role in the developing a nation. However, the system is characterized by multipath propagation effects that lead to signal fluctuation at the receiver, thereby degrading the performance of the system. Maximal Ratio Combiner (MRC) being used to address this problem is characterized with hardware complexity that results in long processing time. Therefore, in this paper enhancement of MRC with closed form expression over Log-Normal fading channel is carried out using Firefly Algorithm (FA) at the receiver. FA create a flashing light in associated with Signal to Noise Ratio (SNR) of the received signal and output the signal with highest SNR. Randomly generated bits used as source data are modulated using M-ary Quadrature Amplitude Modulation (M-QAM) and transmitted over Log-Normal fading channel to generate resultant signals. The resultant signals at varying paths ' $L$ ' $(2,3,4)$ are optimized by $F A$. The outputs of the FA are combined by MRC to obtain the received signal and converted to baseband through demodulation. A mathematical expression using the Probability Density Function (PDF) of Log-Normal fading channel at varying paths ' $L$ ' for Bit Error Rate (BER) is also derived. The technique is simulated using MATLAB 7.4. The performance is evaluated using Signal-to-Noise Ratio (SNR), Bit Error Rate (BER) and Processing Time (PT). The result shows that, the enhanced MRC (eMRC) gave lower BER values when compared with conventional MRC with reduction in hardware complexity. The eMRC carried out can be used to enhance the performance of wireless communication system.
\end{abstract}

Keywords: Firefly Algorithm (FA), Maximum Ratio Combiner (MRC), Bit Error Rate (BER), Square Root Raise Cosine (SRRC) filter, log-normal fading channel.

\section{Introduction}

Wireless Communication (WC) is the exchange of information between two or more users that are not connected by an electrical conductor [3]. The system used to process the information to be transmitted through space consists of transmitter, receiver and the channel through which signal propagates. Transmitter encode, modulate and filter the information to be transmitted before propagating through the space. The signal processing techniques varies the parameters of the carrier signal in accordance with 
Abolade, R.O., Ojo, S.I, Salami, H.T., Adeyemo, Z.K., Akintoye, N.O.; Enhancement of Maximal Ratio Combiner over LogNormal Fading Channel using Firefly Algorithm, Transactions on Networks and Communications, Volume 7 No. 2, April (2019); pp: 1-13

the encoded data for efficient transmission over the wireless channel. In wireless communication, wave propagation refers to the behaviour of signal when they are propagated from transmitter to receiver. In the course of propagation, signals are mainly affected by three different propagation mechanisms such as reflection, diffraction and scattering that result in signal fading [7]. Fading is the variation in signal amplitude over time and frequency. It is either due to multipath propagation, referred to as multi-path fading, or to shadowing from obstacles, referred to as shadow fading [8]. Multipath propagation is a phenomenon that occurs when a transmitted signal propagates in multiples as a result of obstruction in terrestrial environment that results in radio signals reaching the receiving antenna by two or more paths. Transmitted signal resulted into varying signal with fluctuation in strength at the receiver due to multipath fading. The received signal varies with time, geographical position or radio frequency, causing poor reception of the signal and degrades the performance of the system. Thus, fading compensation is required to mitigate this effect [9].

The effects of multipath fading in a WC are addressed using Diversity Combining Techniques (DCTs). The main purpose of DCTs is to combine the independent paths to reduce the effects of fading $[10,11,12$, 13]. Existing Diversity Combining Techniques (DCT) used to solve the multipath propagation effects are Maximal Ratio Combining (MRC) where each path is weighted, cophased and sum all the paths to enhance the signal strength. The second is Equal Gain Combining (EGC) which is similar to MRC except that each signal branch is weighted with the same factor that is, the branches are multiplied by unity, irrespective of the signal amplitude. The third is Selection Combining (SC) which is simply the process of selecting the branch having the highest Signal to Noise Ratio (SNR). Among the existing DCTs, MRC and EGC provide better performance than other combining techniques but at the expense of hardware complexity. The complexity of MRC receivers depends on the number of diversity paths combined and this can be quite high, especially for multipath diversity of wideband Code Division Multiple Access (CDMA) signals. In this paper an enhanced MRC technique is carried out to reduce the complexity of MRC. The statistical effects of multipath phenomenon in WC followed different fading distributions such as Log-Normal, Weibull, Rayleigh and Rician distributions. In this paper, Log-Normal distribution is used due to its ability to model both inside and outside environment.

\section{Log-Normal Distribution}

Vegetation and foliage are significant factors that cause scattering and absorption of radio waves. As a result, the received signal power varies about the mean power predicted by reduction in signal strength. Log-Normal distribution is applicable in modelling and evaluation of the cellular mobile networks coverage. The fading over large distances causes random fluctuations in the mean signal power and this fluctuation are Log-Normally distributed. This distribution often gives the best fit to outdoor and indoor mobile multipath propagation. The Probability Density Function (PDF) $P_{r}(\gamma)$ of the received signals envelope of this distribution is given by [14] as

$$
P_{r}(\gamma)=\frac{\xi}{(2 \pi \gamma \sigma)^{\frac{1}{2}}} \exp \left(-\frac{\left(10 \log _{10} \gamma-\mu\right)}{2 \sigma^{2}}\right)^{2}
$$

where: $\gamma$ is the amplitude of the received signal

$\mu$ and $\sigma$ are the mean and the standard deviation of $10 \log _{10} \gamma$ respectively 


$$
\xi=10 / \ln 10=4.3429 \text {. }
$$

\section{Maximal Ratio Combining Technique}

Maximal Ratio Combiner (MRC) is one of the diversity combining techniques at the receiver to improve the efficient of the wireless system. It is a commonly used technique to improve the performance in a noise communication system where the AWGN and fading are independent amongst the diversity branches [11]. In the MRC technique, signals from different diversity branches are co-phased and weighted before summing. The weight is chosen as proportional to the respective signals level for maximizing the combined Signal-to-Noise Ratio (SNR). MRC is the optimal combining scheme regardless of fading distribution but at the expense of complexity due to knowledge of all channel fading parameters [12]. Since knowledge of channel fading amplitudes is needed for MRC, this scheme is used in conjunction with unequal energy signals, such as M-ary Quadrature Amplitude Modulation (M-QAM) or any other amplitude and phase modulations. Figure 1 shows the block diagram of MRC. It consists of three receiving antennas which receive the corrupted signal through the space. The signal passes through the separate channel estimator, matched filter and combine before detection. The Probability Density Function (PDF) of the MRC $P D F_{M R C}$ output is given by $[14,15]$ as

$$
P D F_{M R C}=\frac{1}{(L-1) !} \frac{\gamma_{t}^{L-1}}{\gamma_{a v}^{L}} \exp \left(-\frac{\gamma_{t}}{\gamma_{a v}}\right)
$$

where: $\gamma_{a v}$ is the average SNR at each

diversity element,

$\gamma_{t}$ is the SNR ratio at the output of the MRC and $L$ is the number of path

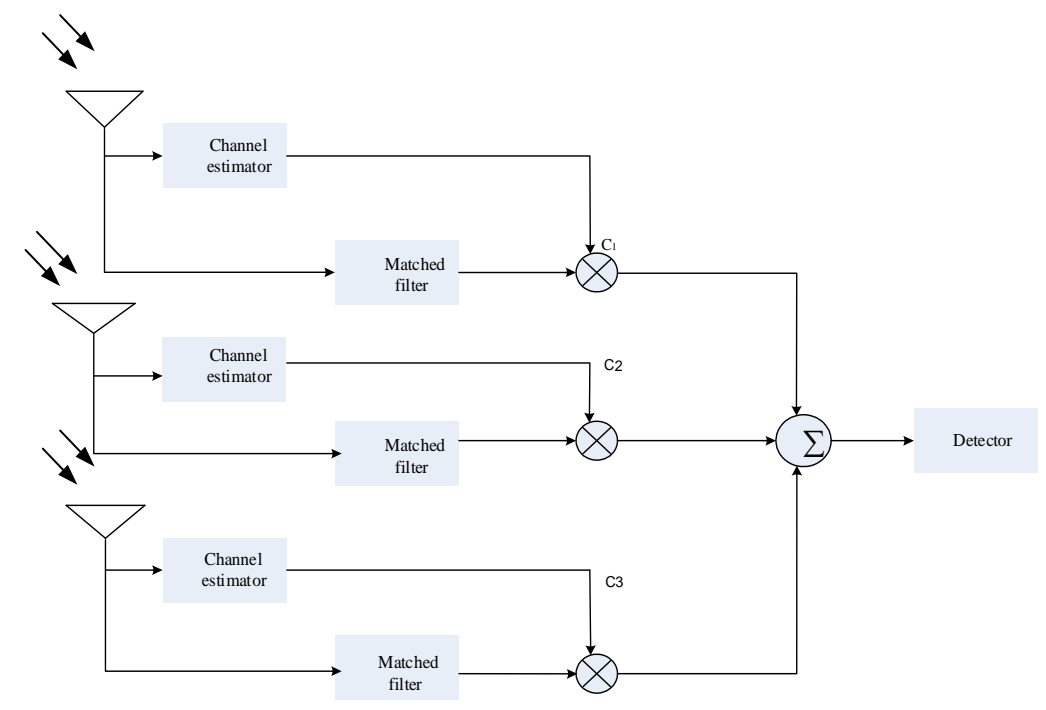

Fig. 1: Block diagram of Maximum Ratio Combining

\section{Bit Error Rate (BER)}

Bit Error Rate (BER) is defined as the ratio of erroneous to total number of transmitted bits. BER is one of the most important criterion to evaluate system performance. The difficulty in determine average BER arises from the fact that the conditional BER is, in general, a non-linear function of the instantaneous SNR. 
Abolade, R.O., Ojo, S.I, Salami, H.T., Adeyemo, Z.K., Akintoye, N.O.; Enhancement of Maximal Ratio Combiner over LogNormal Fading Channel using Firefly Algorithm, Transactions on Networks and Communications, Volume 7 No. 2, April (2019); pp: 1-13

The non-linearity is a function of the modulation detection scheme [5]. Average BER is calculated by obtains the error probability over the PDF of the SNR, the expression for BER is given by Rappaport [5] as

$$
P_{b}(E)=\int_{o}^{\infty} P_{b}(E / \gamma) P_{\gamma}(\gamma) d \gamma
$$

where: $P_{b}(E / \gamma)$ is the conditional error probability. $P_{\gamma}(\gamma)$ is the PDF of the SNR

$E$ is signal per bit or signal per symbol

$\gamma$ is the received SNR

\section{Quadrature Amplitude Modulation (QAM) Signalling Scheme}

Quadrature Amplitude Modulation (QAM) is a type of digital modulation that modifies the phase and amplitude of the transmitted signal simultaneously. QAM offers increased in data throughput and spectral efficiency which is accomplished by varying the amplitude of two sinusoidal waveforms that are in quadrature to each other. It allows effective transmission of two channels at the same frequency and thereby doubling the rate at which the data is transmitted. QAM is regarded as a non-constant envelope schemes that can achieve higher bandwidth efficiency and is widely used in system designed for telephone channels. A signal representation of a signal ' $S_{i}(t)$ ' modulate by QAM is given by [5] as

$$
S_{i}(t)=A_{i} \operatorname{Cos}\left(2 \pi f_{c} t+\theta_{i}\right)
$$

where: $S_{i}(t)$ is the transmitted signal

$A_{i}$ is the signal amplitude

$\theta_{i}$ is the phase of the signal

$f_{c}$ is the carrier frequency and

\section{Firefly Algorithm}

Fireflies (lightning bugs) belong to a family of insects that are capable of producing natural light to attract a mate or prey. There are about two thousand lightning bugs species that produce short and rhythmic flashes. These flashes often appear to be in a unique pattern and produce an amazing sight in the tropical areas during summer. The intensity of flashes decreases as the distance increases and thus most lightning bugs communicate up to several hundred meters. Firefly Algorithm (FA) is a stochastic and nature-inspired heuristic algorithm. It is an optimization algorithm that is inspired by social behaviour of fire flies. FA is one of the recent swarm intelligence schemes that is used to solve the hardest optimization problems and is based on the flashing as well as attraction characteristics of the population. The major objective of this algorithm is to obtain the maximum functions, known as objective functions that may be subject to constraints on its variables. The implementation of FA involves the formulation of flashing light, in such a way that it gets associated with the objective function to be optimized. The two important variables in FA are light intensity and attractiveness. Firefly is attracted toward other firefly that has brighter flash than itself and the attractiveness depend on the light intensity. The attractiveness is inversely proportional to the brightness that decreases as distance increases between the fireflies [1, 2]. 
FA is based on three major principles includes: all fireflies are unisex and attracted toward each other regardless of gender, the attractiveness of the fireflies is proportional to the brightness of fireflies and thus the one with less bright will move towards more attractive firefly and the light intensity of a firefly depend on the type of function to be optimized. In practice, the brightness of firefly is directly proportional to the value of the objective function. In each iterative step, FA computes the brightness and the relative attractiveness of each lightning bugs. Based on these values, the positions of the fireflies are updated and after a sufficient number of iterations, all fireflies converge to the best possible position on the search space [16]. The dimension of the search space is equal to the number of optimizing parameters in the given problem and the algorithm works based on global communications among the fireflies $[17,18,19]$. For a given medium with a fixed light absorption coefficient ' $\gamma$ ', the light intensity $\mathrm{I}(r)$ is given by [1] as

where: $\mathrm{I}_{0}$ is the initial light intensity

$$
\mathrm{I}(r)=\mathrm{I}_{0} \exp \left(-\gamma r^{2}\right)
$$

$r$ is the distance between fireflies

The attractiveness ' $\beta$ ' of a firefly is given by [2] as

$$
\beta=\beta_{0} \exp \left(-\gamma r^{n}\right) \quad n \geq 1
$$

where: $\beta_{0}$ is the attractiveness at $r=0$

$\gamma$ is the light absorption coefficient.

$\mathrm{n}$ is the number of iterations

For two fireflies $i$ and $j$ situated at $x_{i}$ and $x_{j}$ respectively, the distance $r_{i j}$ between them is given by [9] as

$$
r_{i j}=\left(\sum_{k=1}^{d}\left(x_{i k}-x_{j k}\right)^{2}\right)^{\frac{1}{2}}
$$

where: $\quad x_{i k}$ is the $k^{\text {th }}$ component of the spatial

coordinate $x_{i}$

$x_{j k}$ is the $k^{\text {th }}$ component of the spatial coordinate $x_{j}$

$d$ is the number of distance and if $d=2$, Equation (8) becomes

$$
r_{i j}=\left(\left(x_{i 1}-x_{j 1}\right)^{2}+\left(x_{i 2}-x_{j 2}\right)^{2}\right)^{\frac{1}{2}}
$$

The movement of a firefly $i$ when attracted to another brighter firefly $j$ is given by [18] as

$$
x_{i}=x_{i}+\beta_{0} \exp \left(-\gamma r^{2}\right)\left(x_{j}-x_{i}\right)+\quad \alpha(\operatorname{rand}-0.5)
$$

where: the first term is the present

position of a firefly,

the second term defines a firefly's attractiveness to light intensity as seen by the adjacent firefly and the third term stands for the random movement of a firefly if no brighter firefly is left.

the coefficient $\alpha$ is a randomization parameter and $r$ and is a random number generator that is uniformly distributed over the space.

\section{Methodology}

Development of an enhanced Maximum Ratio Combining (MRC) technique over Log-Normal fading channels is carried out using Firefly Algorithm (FA). The system model is developed using FA to select the strong paths among the received signals before applied MRC. Closed form expression of PDF for the received signal is derived for the enhanced technique to measure performance of the system. Extensive 
Abolade, R.O., Ojo, S.I, Salami, H.T., Adeyemo, Z.K., Akintoye, N.O.; Enhancement of Maximal Ratio Combiner over LogNormal Fading Channel using Firefly Algorithm, Transactions on Networks and Communications, Volume 7 No. 2, April (2019); pp: 1-13

simulations using MATLAB 7.2 simulation software is used to investigate the performance of the technique. The model is evaluated using Bit Error Rate (BER) and Processing Time (PT) by compared with the conventional MRC technique.

\subsection{Development of an enhanced Maximum Ratio Combining (eMRC)}

The faded signal through Log-Normal fading channel resulted to multipath and Firefly Algorithm (FA) received the multipath signals as shown in Figure 2. FA scan through multipath to select the best paths that is, the paths with highest power. The paths with highest SNR which is the output of FA serves as input to conventional MRC. These branches of signal are co-phased, weigh by different weight and sum up.

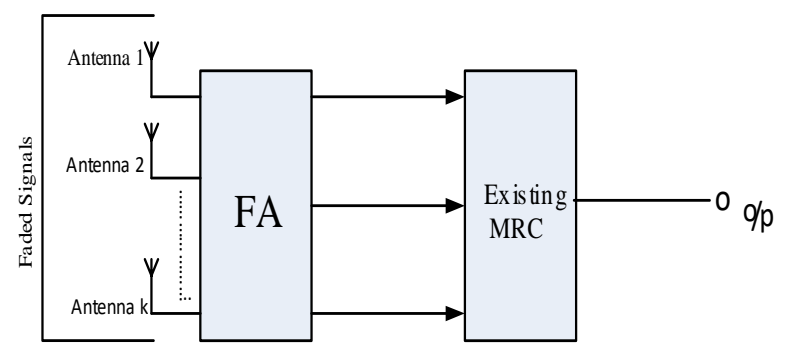

Fig. 2: Block Diagram of an optimized Maximum Ratio Combining

\subsection{Procedure of Firefly Algorithm for the System}

Firefly Algorithm (FA) is a meta-heuristic, nature inspired optimization algorithm that work based on the social flashing behaviours of fireflies. Formulation of flashing light is done based on the objective function that is, the received signals. The parameter considered in the optimization of the received signals is Signalto-Noise Ratio (SNR). The purpose of FA is to select signal with highest SNR. Based on this, the FA is initiated and the attractiveness, distance, position movement and fitness value of firefly are calculated using Equation (5). The attractiveness decreases with increase in distance because of the absorption factor. Therefore, the distance of attraction of brightest firefly is calculated using Equation (7). The movement of $\mathrm{i}^{\text {th }}$ firefly towards brighter $\mathrm{j}^{\text {th }}$ firefly is based on the attractiveness and distance between them and this is calculated using Equation (8). If the firefly does not find any brighter firefly, it will move randomly and the above process is repeated until a brighter firefly is found and stopping criterion is achieved. The brightest firefly is the signal with highest SNR (power). 


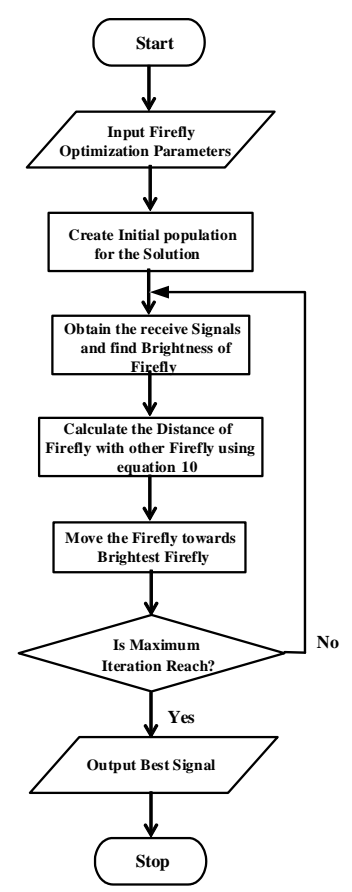

Fig. 3: Flowchart of Firefly Algorithm

\subsection{Probability Density Function (PDF) of the received signal for the System}

Considering a Log-Normal fading channel, the optimized branches which is the output of Firefly Algorithm (FA) is denoted as ' $\gamma_{o p}$ '. The inputs of MRC are outputs of FA ' $\gamma_{o p}$ ' and MRC having L branches, the output $\operatorname{SNR}\left(f_{M R C}(r)\right)$ is given by [20] as

$$
f_{M R C}(r)=\frac{\gamma^{l-1} \exp (-\gamma / \bar{\gamma})}{\bar{\gamma}^{l} \Gamma(l)}
$$

where: $\gamma$ is the instantaneous SNR which is

the output of FA, that is, optimized paths

$\bar{\gamma}$ is the average SNR

$\Gamma(l)$ is the Gamma function

$l$ is the diversity branch

The output SNR for the optimized MRC ' $f_{O M R C}(r)$ ' is given as

$$
f_{\text {OMRC }}(r)=\frac{\gamma_{o p}{ }^{l-1} \exp \left(-^{\gamma_{o p}} / \bar{\gamma}\right)}{\bar{\gamma}^{l} \Gamma(l)}
$$

Probability Density Function (PDF) of output SNR of MRC combiner $P D F_{M R C}$ is given in Equation (2). Substituting Equation (11) into Equation (2) gives

$$
P D F_{M R C}=\frac{1}{(L-1) !} \frac{\frac{\left(\gamma_{o p}{ }^{l-1} \exp \left(-^{\gamma_{o p}} / \bar{\gamma}\right)\right)}{\bar{\gamma}^{l} \Gamma(l)}{ }^{L-1}}{\gamma_{a v}^{L}} \exp \left(-\frac{\frac{\gamma_{o p}{ }^{l-1} \exp \left(-\gamma_{o p} / \bar{\gamma}\right)}{\bar{\gamma}_{\Gamma(l)}}}{\gamma_{a v}}\right) \frac{\gamma_{o p}{ }^{l-1} \exp \left(-{ }^{\gamma_{o p}} / \bar{\gamma}\right)}{\bar{\gamma}^{l} \Gamma(l)}
$$


Abolade, R.O., Ojo, S.I, Salami, H.T., Adeyemo, Z.K., Akintoye, N.O.; Enhancement of Maximal Ratio Combiner over LogNormal Fading Channel using Firefly Algorithm, Transactions on Networks and Communications, Volume 7 No. 2, April (2019); pp: 1-13

The expression for Bit Error Rate ' $P_{b}(E)$ ' is given in Equation (3), but for the optimized MRC, $P_{\gamma}(\gamma)$ in Equation (6) is equal to $P D F_{M R C}(\gamma)$. Therefore, equation (3) becomes

$$
P_{b}(E)=\int_{o}^{\infty} P_{b}(E / \gamma) P D F_{M R C}(\gamma) d \gamma
$$

Substituting Equation (12) into Equation (13) gives

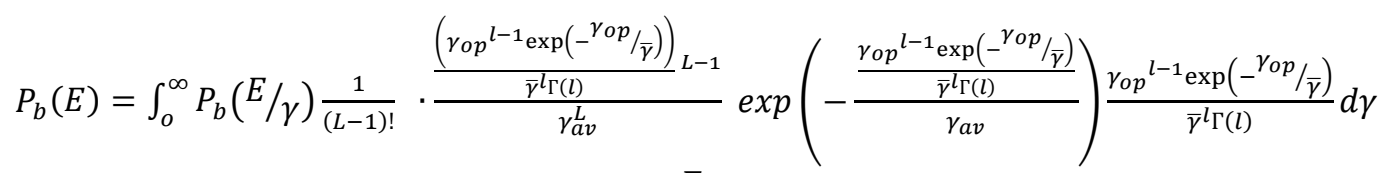

According to [20], conditional error probability $P_{b}(E / \gamma)$ is given as

$$
P_{b}(E / \gamma)=1 / 2 \exp (a \gamma)
$$

where $a=0.5$ for non-coherent modulation

For non-coherent modulation, Equation (15) becomes

$$
P_{b}(E / \gamma)=1 / 2 \exp (0.5 \gamma)
$$

Substituting Equation (16) into Equation (14) gives

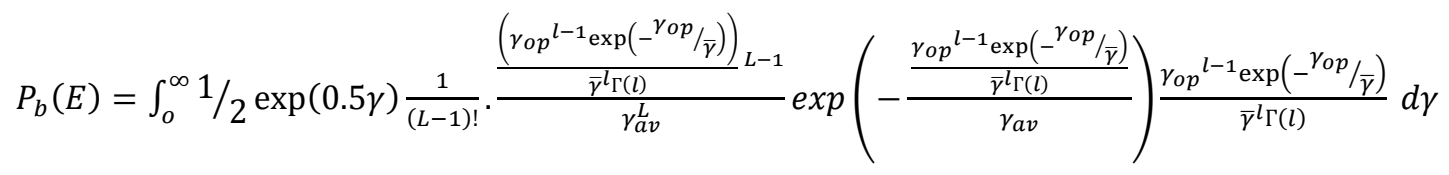

Equation (17) is the Bit Error Rate (BER) for the enhanced MRC.

\section{Simulation Model}

The system model for this work consists of transmitter, Log-Normal fading channel, and the receiver. Data acquisition is from the random integer generator which is available within the MATLAB. The transmitter processes the information signal (randomly generated data) for transmission, by converting the source data into bits, reshaping and modulating with 4 QAM and 16QAM signalling schemes at different trial. The Square-Root Raised Cosine (SRRC) filter is used at the transmitter to reduce the bandwidth of transmitted signal for suitable transmission over the channel without losing the content of the digital data and thereby improving the spectral efficiency. The received signals over Log-Normal fading channel is combined at RF stage using enhanced Maximum Ratio Combining (eMRC). Output of eMRC is then passed through the RF chain, SRRC filter for further processing and finally demodulated with 4QAM and 16QAM demodulator after it has been gray decoded. The received signal is compared with the transmitted signal to compute Bit Error Rate (BER). The complete simulation model for this work is shown in Figure 4 and Flowchart of the process is shown in Figure 5. In the distributions, 1, 2 and 3 represent the Log-Normal fading channel for three difference paths. Table 1 shows the parameters used during simulation. 


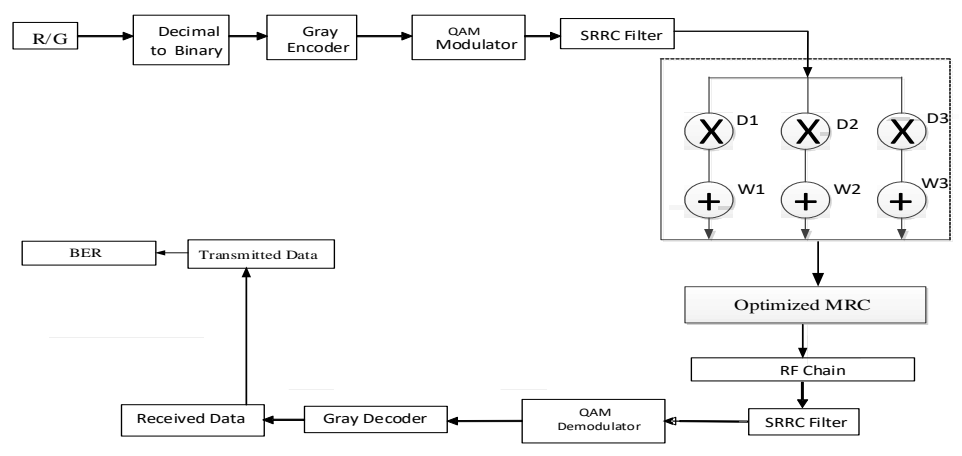

Fig. 4: The system simulation model.

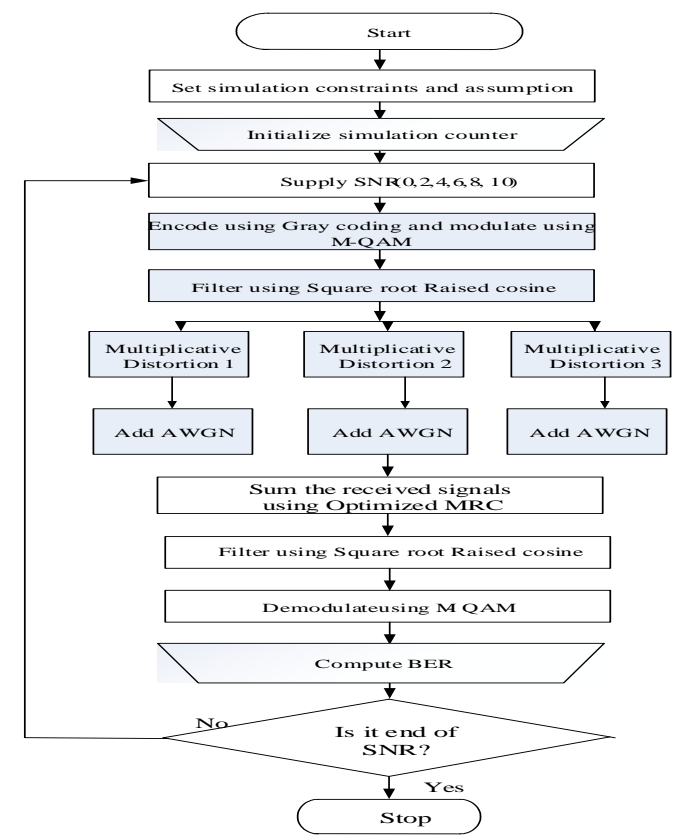

Fig. 5: Flowchart for the system simulation

Table 1: System Simulation Parameters

\begin{tabular}{ll}
\hline Parameter & Specification \\
\hline Modulation schemes & $\begin{array}{l}\text { 4QAM and } \\
\text { 16QAM } \\
\text { Lading }\end{array}$ \\
& $\begin{array}{l}\text { Lading channel } \\
\text { fadmal }\end{array}$ \\
Number of paths & 2,3 and 4 \\
Carrier Frequency & $1800 \mathrm{MHz}$ \\
Noise & AWGN \\
Transmit Filter & Square Root \\
& Raised Cosine \\
Receiver Filter & Square Root \\
& Raised Cosine \\
Roll of factor & 0.25 \\
SNR & $0,2,4 \ldots . . . . ., 10$ \\
Number of symbol (data length) & 10,000 \\
\hline
\end{tabular}




\section{Results and Discussion}

\subsection{Bit Error Rate (BER)}

The values of Bit Error Rate (BER) obtained for both conventional and enhanced MRC are presented in Figs 6 to 11. Fig 6 shows the BER versus SNR for both conventional and enhanced MRC at L of 2 and 4QAM signalling scheme. The BER values obtained for the enhanced MRC are $1.25 e^{-6}, 1.84 e^{-7}, 2.66 e^{-8}$ at SNRs of $4,6,8 \mathrm{~dB}$, respectively as against the conventional MRC with BER values of $2.51 e^{-6}, 3.69 e^{-7}$, $5.33 e^{-8}$ at SNRs of $4,6,8 \mathrm{~dB}$, respectively. Fig 7 shows the BER versus SNR for both conventional and enhanced MRC at $L$ of 2 and 16QAM modulation scheme. At SNR of 2, 4, $6 \mathrm{~dB}$, the BER values obtained for enhanced $\mathrm{MRC}$ are $5.01 e^{-6}, 7.72 e^{-7}, 1.13 e^{-7}$ as against the conventional MRC of $1.00 e^{-5}, 1.54 e^{-6}$, $2.25 e^{-7}$, respectively. Similarly, Fig 8 depicts the BER versus SNR for conventional and enhanced MRC at L of 3 and $4 Q A M$ scheme. It can be deduced that at SNR of 4, 6 and $8 \mathrm{~dB}$, BER values obtained for enhanced MRC are $1.74 e^{-8}, 5.07 e^{-10}, 3.07 e^{-10}$, respectively, as against $3.49 e^{-8}, 1.01 e^{-9}$ and $6.14 e^{-10}$ obtained for conventional MRC. Also, in Fig 9, at L of 3 and 16QAM modulation scheme, BER values obtained for enhanced MRC at SNR of 2, 4, $6 \mathrm{Db}$ are $2.99 e^{-7}, 4.91 e^{-9}$ and $3.95 e^{-10}$, respectively, while $5.99 e^{-7}$, $9.83 e^{-9}$ and $7.91 e^{-10}$ are respectively obtained for conventional MRC. Fig 10 is BER versus SNR for the enhanced and conventional MRC at $L$ of 4 and 4 QAM modulation scheme. It is shown that at SNR of 2, 4 and $6 \mathrm{~dB}$, the $\mathrm{BER}$ obtained for enhanced $\mathrm{MRC}$ are $8.91 e^{-10}, 3.14 e^{-10}$ and $3.97 e^{-10}$, respectively, as against $1.78 e^{-9}, 6.28 e^{-10}$ and $7.94 e^{-10}$, respectively for conventional MRC. Fig 11 shows the BER versus SNR for the enhanced and conventional MRC at $L$ of 4 and 16QAM scheme. The results obtained show that at SNR of 2,4 and $6 \mathrm{~dB}$, the BER obtained are $4.73 e^{-8}, 4.22 e^{-10}$ and $3.60 e^{-10}$, respectively, for the enhanced MRC as against $9.47 e^{-8}, 8.44 e^{-10}$ and $7.20 e^{-10}$ for conventional MRC. The results obtained showed that, enhanced MRC gave a lower BER when compared to conventional MRC and this is due to optimization of the received signals before applied MRC. The results obtained also show that, BER decreases as SNR increases and this is due to increase in signal strength as SNR increases.

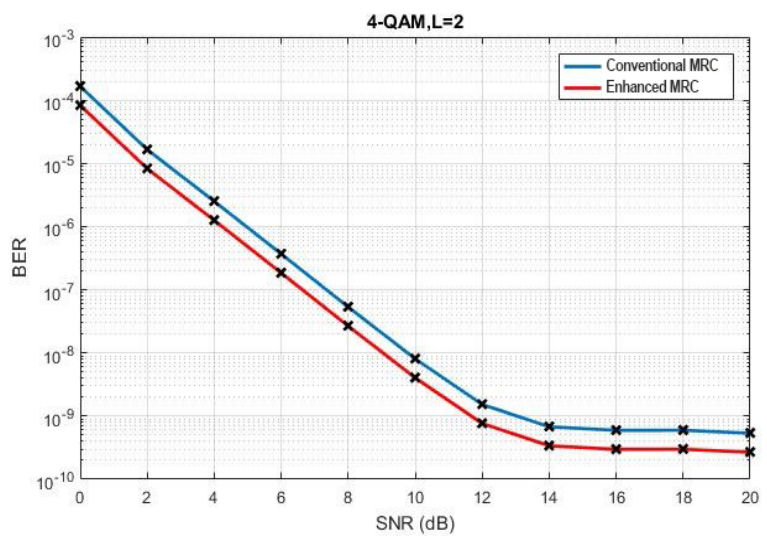

Fig. 6: BER versus SNR for enhanced and conventional MRC at $L=2$ and 4QAM signalling scheme.

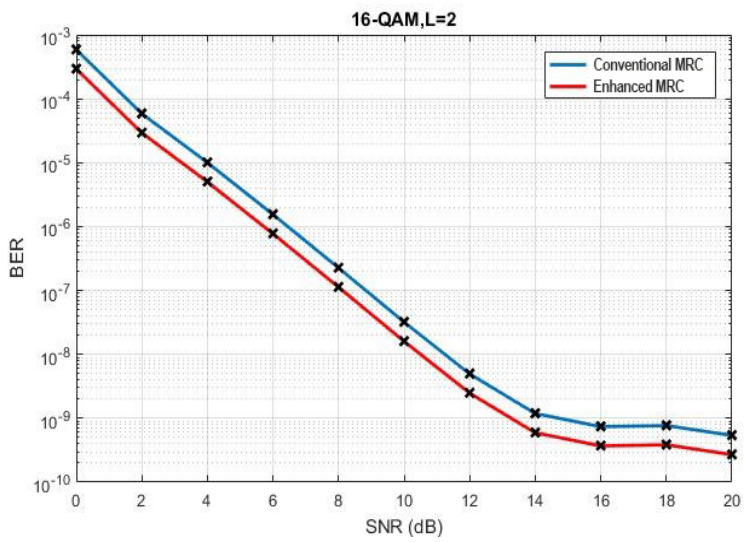

Fig. 7: BER versus SNR for enhanced and conventional MRC at $L=2$ and 16QAM signalling scheme 


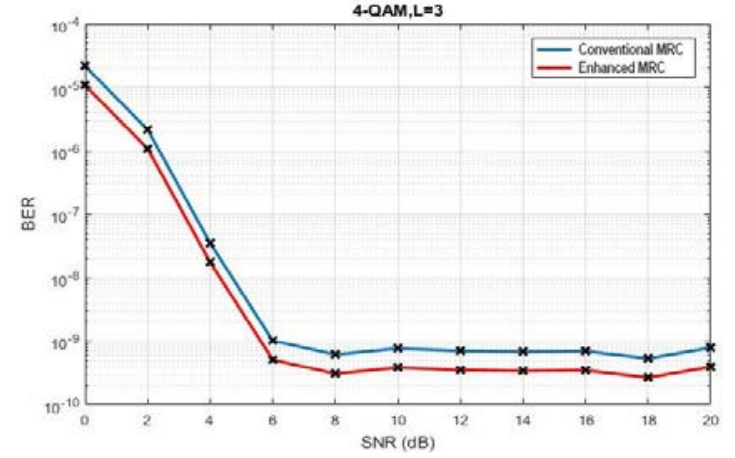

Fig. 8: BER versus SNR for enhanced and conventional MRC at $L=3$ and 4QAM signalling scheme

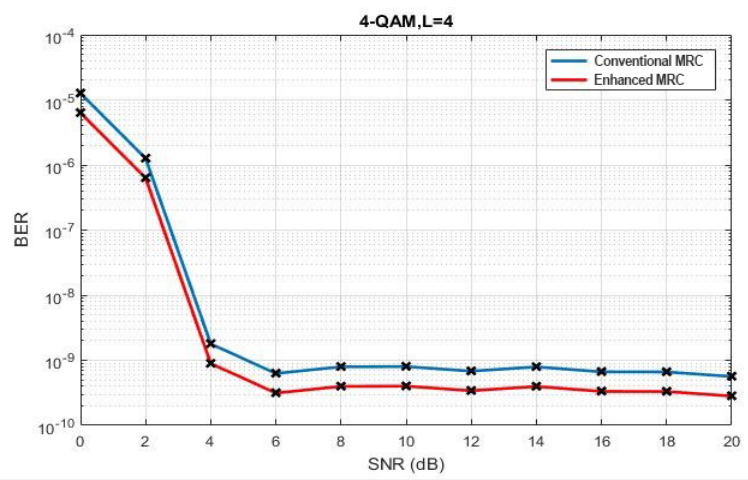

Fig. 10: BER versus SNR for enhanced and conventional MRC at $L=4$ and 4QAM signalling scheme

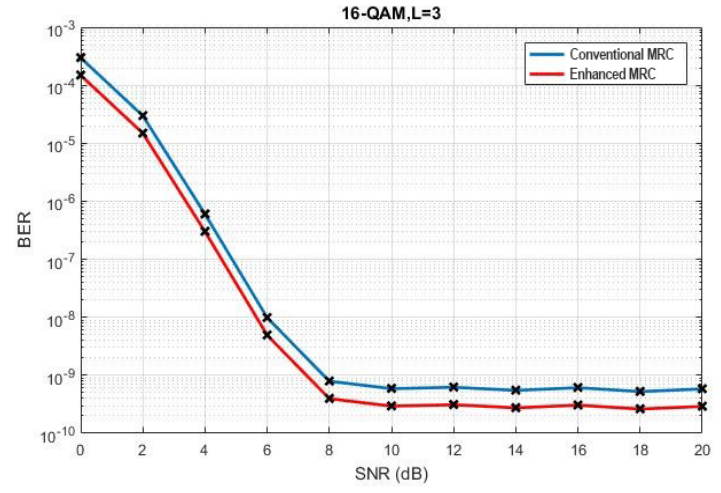

Fig. 9: BER versus SNR for optimized and conventional MRC at $L=3$ and 16QAM signalling scheme

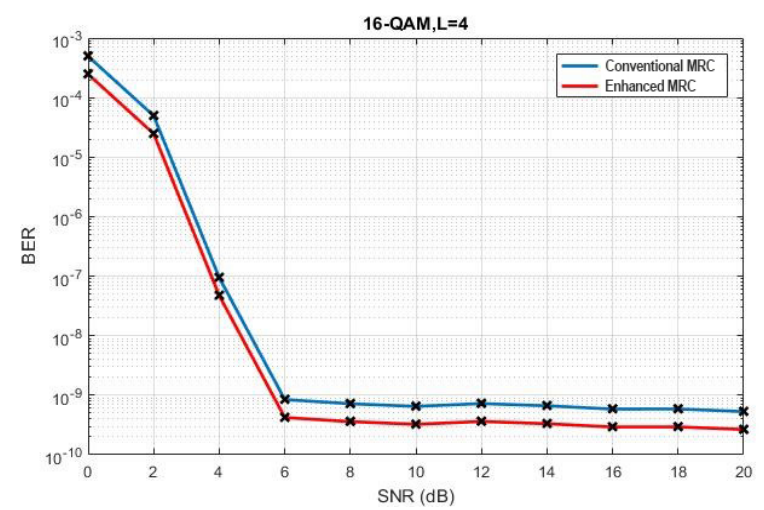

Fig. 11: BER versus SNR for enhanced and conventional MRC at $L=4$ and 16QAM signalling scheme

\subsection{Processing Time for Different Propagation Paths using 4QAM Scheme}

The Processing Time (PT) versus SNR for enhanced and conventional MRC are presented using bar chart in Figs. 12-13 at different paths for 4QAM modulation scheme. Fig. 12 presents the PT with L of 3 using 4QAM scheme over Log-Normal fading channel for enhanced and conventional MRC. At SNR of $6 \mathrm{~dB}$, the PT values obtained are $2.8299 \mathrm{~s}$ and $3.2188 \mathrm{~s}$ for enhanced MRC and conventional MRC, respectively. Fig. 13 shows the PT with L of 4 using 4QAM scheme over Log-Normal fading channel for enhanced and conventional MRC. At SNR of $6 \mathrm{~dB}$, the PT values obtained are $2.9102 \mathrm{~s}$ and $3.1406 \mathrm{~s}$ for enhanced MRC and conventional MRC, respectively. The result is justifiable in that, the hardware complexity for the conventional MRC has been improved using enhanced MRC technique where FA first selects the path with strongest SNR value. The enhancement of MRC is shown from the lower processing time values which indicate better performance. 
Abolade, R.O., Ojo, S.I, Salami, H.T., Adeyemo, Z.K., Akintoye, N.O.; Enhancement of Maximal Ratio Combiner over LogNormal Fading Channel using Firefly Algorithm, Transactions on Networks and Communications, Volume 7 No. 2, April (2019); pp: 1-13

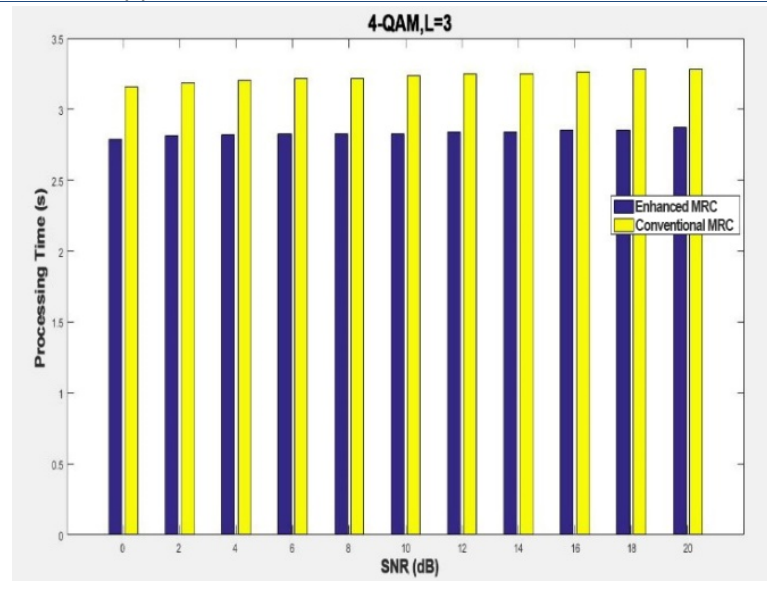

Fig. 12: Processing time versus SNR for $L=3$ using 4QAM signalling scheme over log-normal fading channel

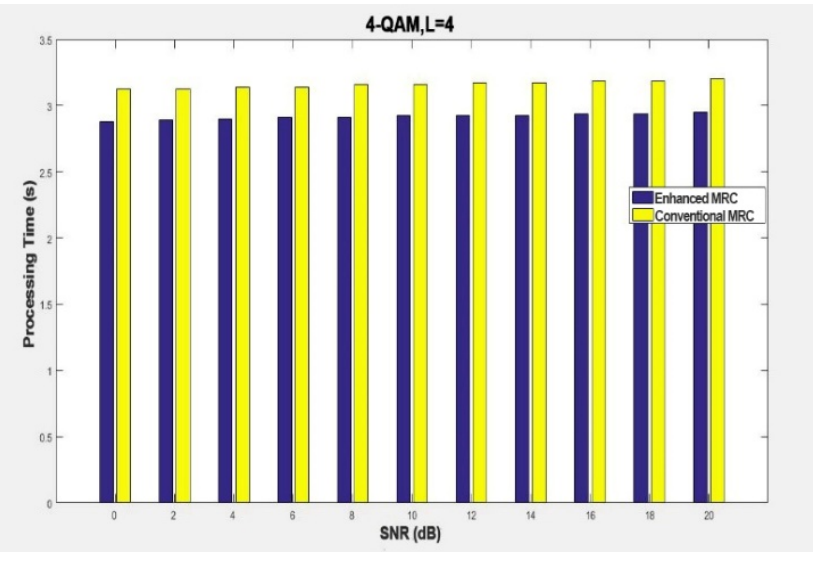

Fig. 13: Processing time versus SNR for $L=4$ using 4QAM signalling scheme over log-normal fading channel

\section{Conclusion}

In this paper, an enhanced Maximum Ratio Combining (MRC) technique over Log-Normal fading channel using FA has been carried out. Mathematical expression for Bit Error Rate (BER) has also been derived using Probability Density Function (PDF). The model has been simulated and evaluated using BER and Processing Time (PT) The results obtained show that the enhanced MRC perform better than conventional $M R C$ due to lower BER and PT values. The performance of the enhanced $M R C$ is as a result of optimization of received signals that select signal with highest power before applying MRC. Therefore, the enhanced MRC has been shown to have a better performance by having a lower BER and PT when compared with the conventional MRC.

\section{REFERENCES}

[1] Adil H., Nishant G. and Divya G. Firefly Algorithm for Unconstrained Optimization. Journal of Computer Engineering. 11(1). PP 75-78 (2013).

[2] Nadhirah A., Mohd A. O., Mohd N. H. and Mohamad H. M. A Review of Firefly Algorithm. ARPN Journal of Engineering and Applied Sciences. 9 (10). PP 1736, (2014).

[3] Jyoteesh, M., Ajay, S.K. and Kaler, R.S. On the performance analysis of wireless receiver using generalized- gamma fading model; Annals Journal of Telecommunications 46(1):147-157. (2009).

[4] Proakis, J. G. Digital Communications Mc Graw-Hill companies, England, pp 348. (2001).

[5] Rappaport, T.S. Wireless Communication Principles and Practice, $2^{\text {nd }}$ edition. Prentice Hall, India, pp 652. (2002).

[6] Stewart, K.A., Labedz, and sohrabi, K. Wideband channel measurements at 900MHZ; IEEE Transaction on Vehicular Technology Conference, Chicago, pp 236-240. (1995) 
[7] Suvarna, P.J. and Vaibhav, S.H. Performance of Maximum Ratio Combining (MRC) MIMO Systems for Rayleigh Fading Channel; International Journal of Scientific and Research Publications, 3(2): 13. (2013).

[8] Adeyemo, Z. K. and Raji T. I. Effects of Diversity combining in mobile Terrestrial Environment; Continental Journal of Engineering Sciences, (5) pp. 27-37. (2010).

[9] Mohamed, S. and Marvin, K.S. Performance of Coherent Receiver with Hybrid SC/MRC Over Nakagami-m Fading Channel; IEEE Transactions on Vehicular Technology 48(4): 1155-1157. (1999).

[10] Goldsmith, A.J. Wireless Communication, first Edition. Cambridge University press. Cambridge, England pp 445. (2005).

[11] Adeyemo, Z.K., Badrudeen and Abolade, R.O. Inter-symbol Interference Distortion Cancellation Using a Modified Maximal Ratio Combiner in Mobile Wireless Communication; Journal of Information Engineering and Application, 3(8): 16-17. (2013).

[12] Rajkumar, G. Performance Evaluation of Maximum Ratio Combining Scheme in WCDMA System for different Modulation; International Journal of Emerging Technologies in Engineering Research 4(5):240-242. (2016).

[13] Abolade, R.O. and Adeyemo Z.K. Spectral Efficiency Analysis for MIMO Beamforming Using Adaptive Modulation, Journal of Theoretical \& Applied Information Technology 49(1): 79-85. (2013).

[14] Simon, M.K. and Alouini, M.S. Digital Communication over fading channels. John Wiley \& Sons. Inc. Hoboken, New Jersey, pp 937. (2005).

[15] Stuber, G.L. Principles of Mobile Communications, Second Edition. Kluwer Academic Publishers New York, pp 771. (2002).

[16] Kumar, B.V. and Srikanth, N.V. BAT Algorithm and Firefly Algorithm for improving dynamic stability of power systems using UPFC. International Journal of electrical engineering and informatics, 8(1), 164-187. (2016).

[17] Ubankar, A.J., Balande, U.T. and Seth, P.D. Performance evaluation of firefly algorithm with variation in sorting for non -linear benchmark problems. AIP conference proceeding, American Institute of Physics, 9(8): 1-9. (2017).

[18] Arora, S. and Singh, S. The Firefly optimization algorithm: convergence analysis and parameter selection. International Journal of Computer Application, 69(3): 48-52 (2013).

[19] Xin-She, Y. and He, X. Firefly algorithm: recent advances and application. International journal of swarm intelligence, 1(1): 36-50 (2013)

[20] Hima, P.V and Seema, P. Performance Analysis of Hybrid MRC/EGC Diversity Combining Technique over AWGN channel; IOSR Journal of Electronics and Communication Engineering, 42(2):25-26. (2016). 